\title{
Cold Relativistic Wavebreaking Threshold of Two-Dimensional Plasma Waves
}

\author{
J. K. Kim, and D. Umstadter \\ Center for Ultrafast Optical Science, University of Michigan, Ann Arbor, MI 48109 \\ July 28, 1998
}

\begin{abstract}
The two-dimensional wave-breaking of relativistic plasma waves driven by a ultrashort high-power lasers, is described within a framework of cold 2-D fluid theory. It is shown that the transverse nonlinearity of the plasma wave results in temporally increasing transverse plasma oscillation in the wake of the laser pulse, inevitably inducing wave-breaking below the 1-D threshold. A condition for wavebreaking is obtained and evaluated. A preformed density channel is found to partially cancel the effect and increase the length of wakefield that survives before wavebreaking occurs.
\end{abstract}

\section{INTRODUCTION}

There has been recently much interest in the propagation of ultrashort high intensity laser pulse propagation through a plasma thanks to the proliferation of compact tabletop terawatt laser systems [1], and applications to laser accelerator of electrons, fast ignitor fusion, $\mathrm{X}$-ray lasers and laboratory astrophysics. When a terawatt laser pulse propagates through an underdense plasma, it generates a relativistic longitudinal plasma wave, so called wakefield, trailing behind the laser pulse. Considering that the field gradient obtainable from the wakefield $(>1 \mathrm{GeV} / \mathrm{cm})$ is enormous in amplitude and the wakefield itself is traveling with the speed very close to $c$, the wakefield can serve as an extraordinary accelerating structure, as was first suggested by Tajima and Dawson [2]. Charged particles, if trapped, can be accelerated until they are eventually dephased with respect to the accelerating field gradient by outrunning the wave.

However, it is a well-known fact that the plasma wave breaks if the wave amplitude is very large. At this electron plasma wave-breaking(epwb) amplitude, the excursion distance of electrons in the wave becomes comparable to the plasma wavelength, thus enabling those electrons to penetrate the acceleration phase of the wakefield, called the separatrix. Once in the separatrix, the electrons gain a net longitudinal momentum consuming all the wave energy and destroying the

CP472, Advanced Accelerator Concepts: Eighth Workshop,

edited by W. Lawson, C. Bellamy, and D. Brosius

(C) 1999 The American Institute of Physics 1-56396-889-4/99/\$15.00 
wake. Thus, epwb leads to bulk production of energetic electrons and, if controlled [3], could serve as a useful technique for an efficient plasma cathode. However, due to the inherently catastrophic nature of uncontrolled epwb, it cannot provide the mono-energetic beam, required for FELs and colliders. For applications such as laser-plasma accelerators, which require quasi-static plasma wave oscillations, epwb sets an upper limit on the largest field gradient extractable from the wake and thus should be avoided in order to maintain a constant peak-amplitude wake structure rather than exponentially decaying one. If, as we will show, epwb inevitably occurs at some distance behind the laser pulse, then it is crucial to know where, since it may be possible to drive the wave back down before epwb occurs. This information is also important because the maximum length of the plasma wave that survives before the onset of epwb also determines the maximum electron current that can be usefully produced by a wakefield.

Much theoretical work has been performed on the subject of epwb of a plasma wave in 1-D geometry. Work on a nonrelativistic cold epwb amplitude was first carried out by Dawson [4], which can be written as $E_{w b}=m c \omega_{p} / e$, where $\omega_{p}=\left(4 \pi e^{2} n_{e 0} / m_{e}\right)^{1 / 2}$ is electron plasma frequency and $n_{e 0}$ is the ambient electron density. The correction on the amplitude including a finite temparature was given by Coffey [5]. It was followed by the 1-D cold relativistic epwb amplitude as $E_{w b}=\left(m_{e} c \omega_{p} / e\right) \sqrt{2\left(\gamma_{\phi}-1\right)}[6]$, where $\gamma_{\phi}$ is the relativistic factor of the plasma wave. Katsouleas et. al. integrated the previous results into a single theory of relativistc warm epwb amplitude in 1-D geometry [7]. Rosenzweig claimed a different answer from Katsouleas' solution, which was eventually resolved, to be unified into a single description by Sheng [8]. All these expressions can be obtained either by evaluating the break point of 1-D relativistic fluid theory $[6,9]$, or by analysis of plasma oscillations of a single electron around an equilibrium point [10]. In as much as it is solely determined by $\gamma_{\phi}$ associated only with longitudinal characteristics of the wakefield, we call it longitudinal wave-breaking. However, all of these works concern only 1-D plasmas. Accordingly, it is inadequate to apply these conditions to 2-D epwb phenomena.

Wave-breaking in the multi-dimensional case is much more complicated than in 1-D, due to the coupling between the transverse and longitudinal plasma oscillation [4]. Transverse variation of the 1-D nonlinearity of a plasma wave results in many interesting aspects of 2-D wakefield such as horse-shoe-shaped wakefield phase front [11]. Due to the nonlinear plasma freqency $\Omega_{p}$, which is smaller than $\omega_{p}$ on the propagation axis of the pump, the phase front of the wakefield experiences increasing distortion and the wake is shown to break after a number of plasma periods behind the laser pulse [12]. This new type of epwb is called transverse wave-breaking. Bulanov et. al. first presented an estimate for the number of unbroken wakefield "buckets" by two-dimensional analysis on the self-intersection of electron trajectories [12]. Their analysis was based on the study of electron fluid oscillation perpendicular to the phase front of the wakefield by means of catastrophic theory. It however explains transverse epwb only qualitatively with the fixed ex- 
cursion distance of an electron fluid element perpendicular to the phase front. For this reason, their expressions of the amplitude and the bucket where epwb takes place for a given plasma channel may not be accurate. The fluid description of the wakefield provides a more solid and quantitative analysis of the epwb condition for a 2-D plasma wave than does a single particle analysis. Since the 1-D epwb amplitude was obtained from both fluid and single particle analysis, it is expected that a generalized 2-D epwb condition can be arrived at with a set of relativistic fluid equations.

We present here such a fluid analysis (with finite $\gamma_{\phi}$ ) of the 2-D epwb process in order to derive a criterion for the epwb condition. We then use our epwb condition to determine how long a 2-D wave will survive without breaking for a given wave amplitude. For the sake of simplicity, we neglect all competing mechanisms, such as the modulational instability, which can damp the wave on the timescale of several ion periods. Also for the sake of simplicity, the plasma wave is assumed to be excited by a single short-pulse laser (called pump). If the pulse width is on the order of the plasma wavelength, the wake is driven resonantly to very large amplitude prescribed by $E_{\max }(\mathrm{GeV} / \mathrm{m}) \approx 3.8 \times 10^{-8} n_{0}^{1 / 2}\left(\mathrm{~cm}^{-3}\right) a_{0}^{2} /\left(1+a_{0}^{2} / 2\right)^{1 / 2}[13]$, while the transverse extent of the channel linearly scales with the laser spot radius $r_{0} . a_{0}$ is the amplitude of the normalized vector potential of the pump.

For a more quantitative characterization of epwb in 2-D fluid theory, it is crucial to understand the transverse nonlinearity of the relativistic plasma wave as well as the longitudinal one. It has been pointed out [12] that for an unguided wakefield accelerator, the change of $\Omega_{p}$ across the channel causes the reduction of the curvature radius of the phase front eventually breaking the wave when the electron displacement becomes larger than the curvature radius. In the longitudinal direction appears the nonlinearity of the sawtooth-shaped oscillation of the wakefield gradient, in which the maximum acceleration and deceleration phase are so spatially close to each other that electrons in the deceleration phase are easily accessible by the acceleration phase via thermal velocity fluctuations. If $r_{0} \gtrsim \lambda_{p}$ where $\lambda_{p}$ is the plasma wavelength, the excursion distance of the transverse plasma oscillation is small compared to the longitudinal one, and thus can be neglected. With this assumption, the approximation that the transverse shape of the plasma wave follows the transverse intensity profile of the pump can be justified.

\section{WAVEBREAKING THRESHOLD}

We start from considering the self-consistent set of 2-D relativistic cold fluid equations coupled to Maxwell's equations with the choice of Gaussian gauge $\nabla \cdot \mathbf{a}=$ 0 ,

$$
\left(\nabla_{\perp}^{2}+\frac{1}{\gamma_{g}^{2}} \frac{\partial^{2}}{\partial \zeta^{2}}\right) \mathbf{a}=k_{p}^{2} \eta_{e} \mathbf{u}-\beta_{g} \nabla \frac{\partial \phi}{\partial \zeta}
$$




$$
\begin{array}{r}
\left(\nabla_{\perp}^{2}+\frac{\partial^{2}}{\partial \zeta^{2}}\right) \phi=k_{p}^{2}\left(\gamma \eta_{e}-\eta_{i}\right) \\
-\beta_{g} \frac{\partial}{\partial \zeta}(\mathbf{u}-\mathbf{a})=\nabla(\phi-\gamma) \\
-\beta_{g} \frac{\partial}{\partial \zeta}\left(\eta_{e} \gamma\right)+\nabla \cdot\left(\eta_{e} \mathbf{u}\right)=0
\end{array}
$$

For our convenience, we denote the perpendicular direction by $r$ and the longitudinal direction by $\zeta$. Therefore, $\nabla_{r} \equiv \nabla_{\perp}$. Normalized scalar and vector potential are, $\mathbf{a}=\left(m_{e} c^{2} / e\right) \mathbf{A}$ and $\phi=\left(m_{e} c^{2} / e\right) \Phi$. The group velocity of the laser pulse is $\gamma_{g}=\left(1-\beta_{g}^{2}\right)^{-1 / 2} \sim \gamma_{\phi}, k_{p}=\omega_{p} / c$, and $\gamma m_{e} c^{2}$ the electron energy. $\mathbf{u}=\gamma \beta$ is the normalized momentum of the electron, $\eta_{e}=n_{e} / \gamma n_{e 0}$ normalized electron density, and $\eta_{i}=n_{i} / n_{i 0}$ the normailized ion density of the channel where $n_{i 0}=n_{e 0}$ for a singly-ionized plasma. In order to obtain Eqs. 1-4, the slowly varying envelope approximation, the two-variable expansion, and the quasistatic approximation are used [14]. Cylindrical geometry is also implicitly assumed. With the coordinate transformation $\zeta=z-\beta_{g} c t$, Eqs. 1-4 become

$$
\begin{array}{r}
u_{\zeta}=[\gamma-(1+\psi)] / \beta_{g} \\
\mathbf{u}_{\perp}=\frac{1}{k_{p}^{2} \eta_{e}}\left[\beta_{g} \nabla_{\perp} \frac{\partial \psi}{\partial \zeta}+\frac{1}{\gamma_{g}^{2}}\left(\frac{\partial^{2}}{\partial \zeta^{2}}+\nabla_{\perp} \nabla_{\perp} \cdot\right) \mathbf{a}_{\perp}\right]
\end{array}
$$

where $\gamma=\sqrt{1+u_{\zeta}^{2}+\mathbf{u}_{\perp}^{2}+\mathbf{a}_{L}^{*} \cdot \mathbf{a}_{L} / 2}, \quad \psi=\phi-\beta_{g} a_{z}$, and $\delta=$ $\left(k_{p}^{2} \beta_{g} \gamma_{g}^{2}\right)^{-1}\left(\nabla_{\perp}^{2}+\partial^{2} / \partial \zeta^{2}\right) a_{z} . u_{\zeta}$ and $\mathbf{u}_{\perp}$ are the longitudinal and transverse momentum of the electron fluid element respectively. $\mathbf{a}_{L}$ is the oscillating component of a at the laser frequency. Plugging Eq. 5 and Eq. 6 in $\gamma$ yields $\gamma=$ $(1+\psi) \gamma_{g}^{2}\{1-\sqrt{1-G}\}$, where

$$
G=\frac{(1+\psi)^{2} \gamma_{g}^{2}+\left(1+\mathbf{u}_{\perp}^{2}+\frac{1}{2} \mathbf{a}_{L}^{*} \cdot \mathbf{a}_{L}\right)\left(\gamma_{g}^{2}-1\right)}{(1+\psi)^{2} \gamma_{g}^{4}}
$$

It is noted that all of the above equations converge to the well-known 2-D relativistic fluid equations obtained with the assumption $\beta_{g}=1$ [14]. If $G>1$, the energy of the electron fluid, $\gamma$, becomes imaginary and the fluid theory breaks, i.e., epwb occurs. Physically, the condition $G=1$, where $\gamma=(1+\psi) \gamma_{g}^{2}$, corresponds to infinite electron density in $\mathrm{Eq} .7$. The description of 2-D epwb is thus very similar to the procedures used in the 1-D case. Inside the wakefield after the pump, $\mathbf{a}_{L}=0$. Therefore, the generalized epwb condition of a relativistic plasma wave in 2-D can be written as $G=1$, which is equivalent to

$$
1+\psi=\frac{\sqrt{1+u_{\perp 0}^{2}}}{\gamma_{g}}
$$


where $\mathbf{u}_{\perp 0}=(1+\psi) \nabla_{\perp}(\partial \psi / \partial \zeta) / k_{p}^{2}\left(\eta_{i}+\nabla_{\perp}^{2} \psi / k_{p}^{2}\right)$. The terms in the order of $1 / \gamma_{g}^{2}$ are neglected in the calculation of $u_{\perp 0}$ whereas $\gamma_{g} \gg 1$ is assumed.

Eq. 8 takes a rather simple form to elucidate both longitudinal and transverse epwb in terms of $\psi$ and $u_{\perp 0}$. As $\gamma_{g}$ goes to infinity, $\gamma$ asymptotes to $\left\{1+u_{\perp}^{2}+\frac{1}{2} \mathbf{a}_{L}^{*} \cdot \mathbf{a}_{L}+(1+\psi)^{2}\right\} / 2(1+\psi)$, which is identical to the previous calculation by Feit [14]. Also, if $u_{\perp 0}=0$, which represents a 1-D plasma wave, Eq. 8 states $\psi=\gamma_{g}^{-1}-1 \approx-1$ for $\gamma_{g} \gg 1$. On account that epwb takes place where $\psi$ is near the minimum value $\psi_{\min }$, the expression properly describes longitudinal epwb by a large-amplitude plasma wave. In addition to this, $u_{\perp 0}^{2}$ in the numerator of $G$ is related to transverse epwb.

Since $u_{\perp 0}$ involves the cross derivative with respect to $r$ and $\zeta$, it is proportional to the increase of the curvature of the phase front. As $\psi$ becomes close to $\psi_{\min }$, the increase of $u_{\perp 0}^{2}$ as well as $(1+\psi)^{-2}$ stimulates the break of the wave. It is also remarked that the epwb threshold in 2-D should be smaller than that of the 1-D case; In a 1-D plasma, the threshold is given by $\psi_{\max , w b}=\left(2 \gamma_{g}^{2}-1\right) / \gamma_{g}-1$, which is equal to $\psi_{\min , w b}=\gamma_{g}^{-1}-1$. As $\gamma_{g}^{-1} \longrightarrow \gamma_{g}^{-1} \sqrt{1+u_{\perp 0}^{2}}$, the expression for the threshold for 2-D plasma wave is turned out to be $\left(\psi_{\max , w b}\right)_{2 d}=\left(2 \gamma_{g}^{2}-1-\right.$ $\left.u_{\perp 0}^{2}\right) / \gamma_{g} \sqrt{1+u_{\perp 0}^{2}}-1$. For example, if $u_{\perp 0} \sim 1$, then the threshold is decreased by a factor of $1 / \sqrt{2}$. This suggests that a $2-\mathrm{D}$ plasma wave has the longitudinally varying threshold, which decreases as $\left(1+u_{\perp 0}^{2}\right)^{-1 / 2}$ along the channel axis.

\section{CHANNEL LENGTH CALCULATION}

It will take several or more plasma periods for $u_{\perp 0}$ to grow until the wave breaks by this type of epwb. Estimation of the number of plasma wave oscillations behind the pump, $N_{w b}$, is of particular importance to many applications such as laser plasma accelerators considering that the length of the plasma wave train driven by a laser pulse cannot be greater than $N_{w b}$. To acquire a simple expression for $N_{w b}$, only the case of $r_{0} \gg \lambda_{p}$ is considered, allowing us to depict the 2-D wakefield by a solution to $\partial^{2} x / \partial \zeta^{2}=\left(k_{p}^{2} / 2\right)\left(\gamma_{\perp}^{2} / x^{2}-1\right)[15]$, where $\gamma_{\perp}^{2}=1+a^{2}$ and $x=1+\psi(\zeta, r)$. The formal solution [15] of the differential equation by Esarey et. al. is $k_{p}(\zeta-L)=$ $2\left(x_{0}\right)^{1 / 2} E(\alpha, \rho)$, where $\alpha=\sin ^{-1}\left[\left(x_{0}-x\right) /\left(x_{0}-x_{0}^{-1}\right)\right]^{1 / 2}, \rho=\left(1-1 / x_{0}\right)^{1 / 2}$, and $E(\alpha, \rho)$ is the incomplete Legendre elliptic integral of second kind. $L$ denotes the pulse length of the pump. In this equation, the transverse profile of the maximum potential, $x_{0}$, is assumed to be small compared to $\gamma_{g}$ but larger than unity. By defining a transverse shape function $S(r)$ of the pump, it is further assumed that $x_{0}(r)=1+\psi_{\max } S(r)$, where $\psi_{\max }$ is the maximum value of the potential on the propagation axis and a constant. For example, if the pump is Gaussian in the transverse dimension, the perturbation on the wake potential approximately follows the shape of the pump, i.e., $S(r)=\exp \left(-r^{2} / r_{0}^{2}\right)$. To validify this premise, 1-D and 2-D particle-in-cell (PIC) simulations (TRISTAN) [16] are used. Both 


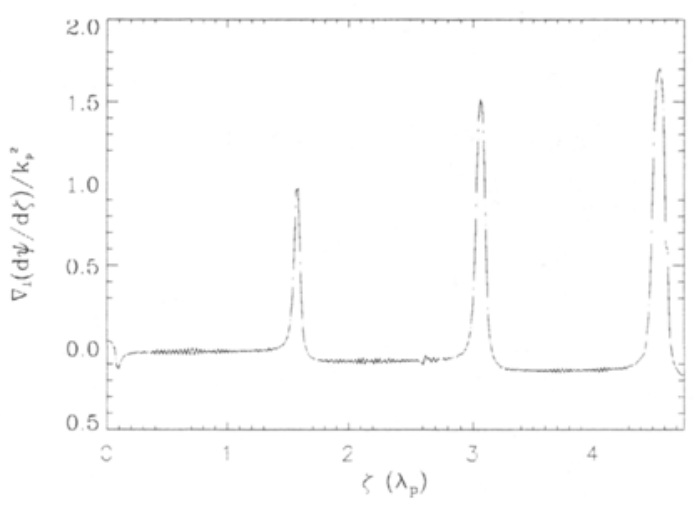

FIGURE 1. Example plot of $\nabla_{\perp}(\partial \psi / \partial \zeta)$ versus $\zeta$ in units of $\lambda_{p}$. At $x \sim x_{0}^{-1}$, where the plasma density has reached maximum, the transverse oscillation also peaks, with an amplitude that increases with $\zeta$. This growth contributes to epwb below the 1-D threshold.

simulations were previously benchmarked with the analytic expression for the 1-D wakefield amplitude and proven to work properly. With $a_{0} \sim 2$ and $r_{0} \gtrsim 2 \lambda_{p}$, a very good agreement on the amplitude of the wakefield is achieved betwen 1-D and 2-D PIC simulations, which justifies the use of the solution to model a moderately two-dimensional plasma wave.

As can be seen from Eq. 8, 2-D epwb critically depends on $u_{\perp 0}$ which is the cause of transverse break of the wave. Hence, for a given value of $\psi$ and a wave-breaking coordinate $P \equiv(\zeta, r), \nabla_{\perp}(\partial \psi / \partial \zeta)$ can be expressed as an explicit function of $\zeta$ and $r$, to be plugged into $u_{\perp 0}$. Denoting $x^{\prime}=\partial x / \partial \zeta$, differentiation of $k_{p}(\zeta-L)=$ $2\left(x_{0}\right)^{1 / 2} E(\alpha, \rho)$ with respect to $r$ gives $\nabla_{\perp} x=\left(F-(\zeta-L) x^{\prime} / 2 x_{0}\right) \nabla_{\perp} x_{0}$, where $F$ is a polynomial funtion of $x$ and $x_{0}$. Integrating the differential equation for the wake potential with respect to $\zeta$ and then differentiating it with respect to $r$, $\nabla_{\perp} x^{\prime}\left(\equiv \nabla_{\perp}(\partial \psi / \partial \zeta)\right)$ is calculated to be

$$
\begin{aligned}
\nabla_{\perp} x^{\prime}= & \frac{k_{p}^{2}}{2 x^{\prime}}\left[\left(1-1 / x_{0}^{2}\right)-\left(1-1 / x^{2}\right) F\right] \nabla_{\perp} x_{0} \\
& +\frac{k_{p}^{2}}{4 x_{0}}\left(1-1 / x^{2}\right)(\zeta-L) \nabla_{\perp} x_{0} .
\end{aligned}
$$

Wave-breaking occurs near $x \sim x_{0}^{-1}$ where the electron density is maximum. Hence, the evaluation of Eq. 9 at $x=x_{0}^{-1}$ yields $\nabla_{\perp} x^{\prime}=-\left(k_{p}^{2} \psi_{\max } / 4\right)\left(x_{0}-x_{0}^{-1}\right)(\zeta-L) \nabla_{\perp} S$. In Fig. $1, \nabla_{\perp}(\partial \psi / \partial \zeta)$ is plotted and the peaks where $x=x_{0}^{-1}$ are shown to linearly increase with $\zeta$.

Plugging Eq. 9 into $u_{\llcorner 0}$, we rewrite Eq. 8 into

$$
\gamma_{g}^{2}=x_{0}^{2}\left[1+\frac{\left(\zeta_{w b}-L\right)^{2}\left|\nabla_{\perp} x_{0}\right|^{2}}{16 \eta_{i}^{2}}\right]
$$




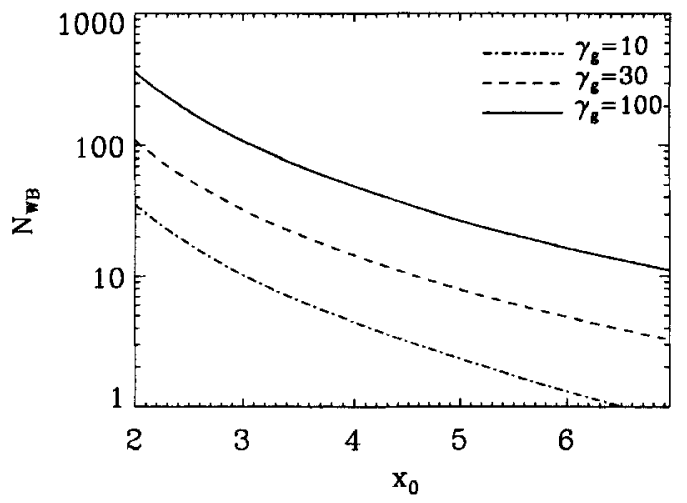

FIGURE 2. $N_{w b}$ in Eq. 11 is plotted versus $x_{0}$, varying $\gamma_{g}$, where $r_{0}=2 \lambda_{p}$ and $\eta_{i}=1$ are used. Decreasing $\gamma_{g}$ reduces $N_{w b}$, effectively limiting the plasma channel length.

where $\zeta_{w b}$ is the $\zeta$-coordinate of $P$. In Eq. 10, the contribution from $\nabla_{\perp}^{2} x$ is neglected since $r_{0} \gg \lambda_{p}$ and $\eta_{i} \gg \nabla_{\perp}^{2} x / k_{p}^{2}$ are assumed. Solving Eq. 10 for $\zeta_{w b}$, we obtain an expression for $N_{w b}$ as

$$
N_{w b} \sim\left(\zeta_{w b}-L\right) / \lambda=\frac{k_{p} \sqrt{\eta_{i}}}{x_{0}^{3 / 2} E_{c}(\rho)} \frac{\sqrt{\gamma_{g}^{2}-x_{0}^{2}}}{\left|\nabla_{\perp} x_{0}\right|},
$$

where $k_{p} \lambda=4 \sqrt{x_{0}} E_{c}(\rho)$ and $E_{c}(\rho)$ is the complete elliptic integral of the second kind. Since it is observed that the maximization of $u_{\perp 0}$ occurs at $r \sim r_{0} / 2$, the estimation of $\left|\nabla_{\perp} x_{0}\right|$ as $x_{0} / r_{0}$ states that $N_{w b}$ is approximately proportional to $\eta_{i}^{1 / 2} r_{0} x_{0}^{-5 / 2}$. For instance, the parameters $\psi_{\max }=3, \eta_{i}=1, r_{0}=6 \lambda_{p}$ and $\gamma_{g}=10$ yield $N_{w b} \sim 7.5$. However, the same parameters with $r_{0}=2 \lambda_{p}$ will give $N_{w b} \sim 2.5$, which represents a case of more tightly focused pump. Eq. 11 is plotted in Fig.2, with $r_{0}=2 \lambda_{p}$. Since $N_{w b}$ scales with $x_{0}^{-5 / 2}$, the drastic decrease of $N_{w b}$ is an unavoidable consequence as $x_{0}$ increases.

Note that $\eta_{i}=\eta_{i}(r)$. Variation of $\eta_{i}$ as a function of $r$ in a preformed density channel used for channel-guiding of the pump, could alter the value of $N_{w b}$, which suggests that the channel guiding can control the number of surviving buckets against epwb. As can be seen in $u_{\perp 0}$ and $N_{w b}$, a strong ion density depression across the plasma channel can be a preventive measure to suppress the transverse break of the wake and extend the length of the plasma wave significantly. The increase of $N_{w b}$ with $\sqrt{\eta_{i}}$ can be interpreted in terms of density-dependent $\gamma_{\phi}$ of the wave. $\gamma_{\phi}=\omega / \omega_{p}$ and $\omega_{p} \propto \sqrt{n_{e}}=\sqrt{n_{i}}$. Subsequently, $\gamma_{\phi}$ is proportional to $\eta_{i}^{-1 / 2}$. It means that the plasma wave propagates faster on the axis than the peripheral region of the channel, thus effectively reducing the phase front distortion of the plasma wave for large $\zeta$. In Fig. 3, $N_{w b}$ is shown as a function of the depth of density depression $\Delta \eta_{d}$ of a preformed plasma density channel. The transverse 


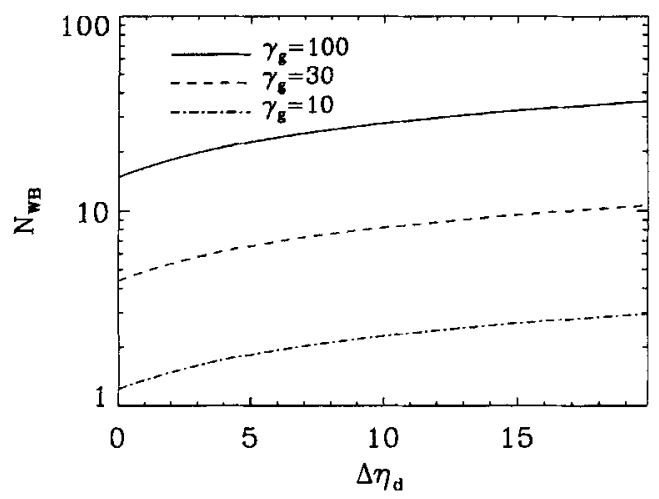

FIGURE 3. $N_{z b b}$ is plotted versus $\Delta \eta_{d}$, varying $\gamma_{g}$, where $r_{0}=2 \lambda_{p}$ and $x_{0}=7$. It is shown that for larger $\Delta \eta_{d}$ and $\gamma_{g}$, the length of a plasma wave train increases.

ion density profile $\eta_{i}=1+\Delta \eta_{d}\left(r^{2} / r_{0}^{2}\right)$ is assumed for $0<r<r_{0}$.

Because $N_{w b}$ scales with $\sqrt{\eta_{i}}$, larger values of $\Delta \eta_{d}$ are preferred in order to increase $N_{w b}$. Conversely, for a given index $j$ for the epwb bucket, the minimum depth of density depression $\Delta \eta_{w b}$ can be calculated to be $4\left\{j^{2} x_{0}^{5} E_{c}^{2} / r_{0}^{2} k_{p}^{2}\left(\gamma_{g}^{2}-x_{0}^{2}\right)-1\right\}$, assuming $r=r_{0} / 2$. Furthermore, a certain level of depression depth is also required for a channel-guiding and it is found to be $\Delta \eta_{c h}=m_{e} c^{2} / \pi e^{2} r_{0}^{2} n_{e 0}$ [17]. Therefore, if $\Delta \eta_{d}>\max \left(\Delta \eta_{w b}, \Delta \eta_{c h}\right)$, it is possible to simultaneously guide the pump in the channel and acquire a plasma wave of which length is approximately $j \lambda_{p}$.

\section{CONCLUSION}

We showed that transverse wave-breaking can be analyzed by standard 2-D cold relativistic fluid theory. It is shown that the transverse nonlinearity of the plasma wave channel results in transverse wave-breaking, which cooperates with longitudinal wave-breaking to break the wave, lowering the break threshold below the 1-D limit. The effect can be at least partially mitigated by use of a density channel. Complete cancellation may be possible, which will be the subject of a future publication.

We gratefully acknowledge support by the Division of High Energy Physics, Office of Energy Research, U. S. Department of Energy, DE-FG02-98ER41071; and the National Science Foundation, STC PHY 8920108. Computing services were provided by the National Partnership for Advanced Computing Infrastructure, PHY $980027 \mathrm{~S}$. 


\section{REFERENCES}

1. M. D. Perry and G. Mourou, Science 264, 917 (1994).

2. T. Tajima and J. M. Dawson, Phys. Rev. Lett. 43, 267 (1979).

3. D. Umstadter, J. K. Kim, and E. Dodd, Phys. Rev. Lett. 76, 2073 (1996).

4. J. M. Dawson, Phys. Rev. 113, 383 (1959).

5. T. P. Coffey, Phys. Fluids 14, 1402 (1971).

6. A. I. Akhiezer and R. V. Polovin, Sov. Phys. JETP 3, 696 (1956).

7. T. Katsouleas and W. B. Mori, Phys. Rev. Lett. 61, 90 (1988).

8. Z. M. Sheng and J. M. ter Vehn, Phys. Plasmas 4, 493 (1997).

9. E. Esarey and M. Pilloff, Phys. Plasmas 2, 1432 (1995).

10. J. Albritton and P. Koch, Phys. Fluids 18, 1136 (1975).

11. S. V. Bulanov, F. Pegoraro, and A. M. Pukhov, Phys. Rev. Lett. 74, 710 (1995).

12. S. V. Bulanov, F. Pegoraro, A. M. Pukhov, and A. S. Sakharov, Phys. Rev. Lett. 78, 4205 (1997).

13. P. Sprangle and E. Esarey, Phys. Fluid. B 4, 2241 (1992).

14. M. D. Feit, J. C. Garrison, and A. M. Rubenchik, Phys. Rev. E 53, 1068 (1996).

15. E. Esarey, P. Sprangle, J. Krall, and A. Ting, IEEE Trans. Plasma Sci. 24, 252 (1996).

16. O. Buneman, T. Neubert, and K. Nishikawa, IEEE Trans. Plasma Sci. 20, 810 (1992).

17. E. Esarey, P. Sprangle, J. Krall, and A. Ting, IEEE J. Quantum Electron. 33, 1879 (1997). 